nen Bereichen leichte Unterschiede im Auflösungsvermögen. Sofern jedoch nicht eine besondere Problemstellung vorliegt, ist das Trennvermögen von käuflichem Cyanogum 41 ausreichend. Von Bedeutung für die
Trennung ist dagegen die Ausnutzung der vollen Laufstrecke sowie das Einwandern der konzentrierten Boratfront des Elektrodenpuffers, die das Ausbilden scharfer schmaler Zonen bedingt.

\title{
Literatur
}

1. Raymond, S. und L. Weintraub, Science (Washington) 130, 711 (1959). - 2. E-C Apparatus. Corp., Techn. Bulletin Nr. 125-B, Literaturübersicht bis 1963, s. auch Brel, H., N. HeimbUrger., D. Kraft, Th. KRANZ und R. Schmidtberger, Behringwerk-Mitt, 43, 1 (1964). - 3. RAYMond, S. und Y. WANG, Analyt. Biochem. (New York) 1, 391 (1960). - 4. RaYMOND, S. und M. NaKAMICHr, Analyt. Biochem. (New York) 3, 23 (1962). - 5. Otr, H. und S. Marinis in Protides of the Biol. Fluids, S. 408. 11th Coll. Brügge
1963, hrsg. von H. Peeters, Elsevier Publ., Amsterdam (1964). 6. Electrophoresis apparatus; E-C Apparatus Corp., 22250 40th Street, University City, Philadelphia 4, PA, USA. - 7. ZwrsLER, O. und H. Brel in: Protides of the Biol. Fluids, S. 433. 12th Coll. Brügge 1964, hrsg. von H. Peeters, Elsevier Publ. Amsterdam (1965). - 8. Mourray, K., Biochim. Biophys. Acta 59, 211 (1962). - 9. Pourrk, M. D., Nature (London) 180, 1477 (1957). - 10. Whrte, M. L., J. phys. chem. 64, 1563 (1960).

Dr. O. Zwisler

Behringwerke AG

335 Marburg/Lahn

\section{Zur Frage der Tagesschwankungen des Serumcholesterins}

\author{
Von CH. StöckiI \\ Aus dem Forscbungslaboratorium der. Neurologischen Universitätsklinik Basel (Direktor: Prof. H. E. Kaeser)
}

(Eingegangen am 9. Juli 1965)

\begin{abstract}
Die Cholesterinbestimmungen wurden mit einer eigenen Ultramikromodifikation der spektrokolorimetrischen Methode nach Huang vorgenommen. Um die Kurve der Tagesschwankungen des Serumcholesterins zu erhalten, wurden pro Versuchsperson, in der Zeit von 8.00-19.00 in stündlichem Abstand 12 Bestimmungen vorgenommen. Das Probandengut bestand aus 15 gesunden Personen, 6 Frauen und 9 Männer zw. 18-56, mit einem Durchschnittsalter von 37 Jahren.

Bei diesen Versuchspersonen konnten rhythmische Tagesschwankungen von gesetzmäßigem Charakter nachgewiesen werden. Darunter gibt es Individuen mit geringen und solche mit beträchtlichen Tagesschwankungen. Nimmt man als Ausmaß der Cholesterin-Tagesschwankung den größten minus den kleinsten Tageswert, so zeigt die labilste Versuchsperson eine solche von $66 \mathrm{mg} / 100 \mathrm{ml}$, die stabilste jedoch nur eine von $6 \mathrm{mg} / 100 \mathrm{~m} /$. Die 3 verglichenen Kurven zeigen übereinstimmend einen morgendlichen Cholesterinanstieg zu einem Hochpunkt, der in die Mittagszeit fällt, und einen anschließenden nachmittäglichen Abfall. Für die Klinik ergibt sich, daß eine optimale Erfassung des Cholesterinspiegels, seiner Schwankungsrichtung und -breite am besten mit einer Drei-Zeit-Messung um 8, 12 und 15 Uhr gewährleistet ist.
\end{abstract}

An ultramicromodification was made to the spectrophotometric method of HuaNG for cholesterol determination. In order to obtain a curve of the daily variation of serum cholesterol, 12 hourly determinations were made on each subject between 8 a. m. and 7 p. m.; 15 healthy persons, 6 women and 9 men, average age 37 (range 18-56) were studied.

In these experiments regular, rythmic, daily variations were found. Some persons showed small and others large daily variations. If the difference between the highest and the lowest daily level is taken as a measure of the daily variation of cholesterol, the most labile subject showed a difference of $66 \mathrm{mg} / \mathrm{ml}$, while the most stable one showed a value of $6 \mathrm{mg} / 100 \mathrm{~m} /$. Each of three comparable curves shows in the morning hours an increase in cholesterol to a maximum during midday which decreases again in the afternoon. For clinical purposes, the optimal determination of the cholesterol level and the direction and extent of its variation is best achieved by three determinations at 8 a. $\mathrm{m}$., noon, and 3 p. m.

In Anbetracht des großen Interesses, welches das Serumcholesterin nicht nur von Seiten der Arterioskleroseforschung, sondern auch in der neurologischen und psychiatrischen Medizin erfahren hat $(1-8,20)$, ist eine neuerliche Abklärung der Frage, ob es sich bei diesem Stoff um einen statischen Parameter handelt, oder ob er gewissen kurzfristigen Tagesschwankungen unterworfen ist, angezeigt.

Aus internistischen Untersuchungen bis etwa 1926 schien hervorzugehen, da $\beta$ die Konzentration des Serumcholesterins beim Gesunden praktisch konstant sei, so- fern nicht durch alimentäre Belastung eine in wenigen Stunden abklingende Erhöhung eintrete. Serienuntersuchungen durch GeORGI von 1926-33 mit 2161 Cholesterin-Bestimmungen bei 456 depressiven Patienten und 21 Gesunden (3) führten jedoch u. a. zur Feststellung, daß physisch und psychisch gesunde Männer keine nennenswerten Schwankungen während Wochen und Monaten aufwiesen, wenn die Blutentnahme zur gleichen Tagesstunde vorgenommen wurde (gesunde Frauen zeigten größere Schwankungen im Praemenstrum). Im Gegensatz zu psychisch Gesunden wurden bei 
Psychosen mit depressivem Charakter leichte bis schwere Hypercholesterinämien festgestellt. - Um diese letzteren Störungen im Cholesterinhaushalt besser zu erfassen, wurden vom gleichen Autor Untersuchungen mit einem Cholesterin-Olivenöl-Belastungstest angestellt (3). Bei endogenen Depressionen zeigte sich in der überwiegenden Mehrzahl - im Gegensatz zu Gesunden - eine sog. „inverse Reaktion“. Statt einer Erhöhung des Serumcholesterins stellte sich ein vorübergehender Abfall ein. Man konnte so praktisch bei jedem endogenen Depressiven eine Störung im Cholesterinstoffwechsel nachweisen. Entweder fand sich ein erhöhter Ausgangswert oder die erwähnte ,inverse Reaktion“. Nachuntersuchungen von EderLe (6), BRUN (7), GiLdeA (8) und SAARNIO (5) haben diese Beobachtungen bestätigt. Die Vermutung eines gewissen und für einzelne Erkrankungen eventuell typischen Tagesrhythmus lag somit nahe. 1938 begann Georgr (3), angeregt durch Arbeiten von G. BRUN, nach autonomen, nicht durch Belastung induzierten Konzentrationsschwankungen zu suchen. Es ergab sich dabei, daß die Cholesterin-Konzentration im Serum - nicht wie noch wenige Jahre zuvor von Sperry, Boyd und Turner $(9,10,11)$ angenommen einen mehr oder weniger konstanten Wert, sondern einen kurvenmäßigen Verlauf erkennen läßt (vgl. Abb., obere Kurve). Bei 2 stündiger Messung zeigt die Kurve im wesentlichen einen Anstieg vormittags, einen Gipfel um die Mittagszeit mit anschließendem Abfall im Verlaufe des Nachmittags. - Eine gleichartige Untersuchung wurde später von LIPPI und Mitarbeitern (12) durchgeführt. Die Bestimmungen erfolgten im Gegensatz zu GeORGr alle 4 Stdn., erstreckten sich dafür aber auch über die Nacht. Untersuchungsgut waren 20 Gesunde (6 Frauen, 14 Männer, Durchschnittsalter: 28 Jahre). Auch hier zeigte sich in den Morgendstunden ein Anstieg gegen den Mittagsgipfel, sowie der nachmittägliche Abfall; diese Abwärtsbewegung kommt in der Kurve der Abbildung bei 15.30 Uhr zum Stillstand. Auf der Strecke $\mathrm{A}-\mathrm{B}-\mathrm{C}$ ergibt sich somit ein analoges Verhalten.

Im folgenden sollen diese interessanten Befunde eine Nachkontrolle bzw. Präzisierung exfahren. Die Bearbeitung dieses Problems trat um so mehr in den Vordergrund, als inzwischen neue Mikromethoden ausgearbeitet wurden, die Fragestellungen erlauben, die mit früheren Methoden kaum zu bewältigen waren, wie etwa der Wunsch nach stündlicher Blutentnahme. Ein weiterer Grund war die Frage, ob der Klinik zur Untersuchung des Kurvenverlaufes bezüglich Konstanz des Merkmals und Größe der eintretenden Veränderungen ein günstigerer, schwankungsarmer Meßbereich vorgeschlagen werden kann als die bisher übliche Zeitspanne zwischen 7-11 Ühr.

\section{Methodik}

\section{Versuchsgut}

15 gesunde, nicht hospitalisierte Personen beiderlei Geschlechts: 6 Frauen. im Alter von 22-46 Jahren (Durchschnitt 34 J.), 9 Männer im Alter von 18-56 Jahren (Durchschnitt $39 \mathrm{~J}$.).

\section{Methode}

$\mathrm{Da}$ die Versuchspersonen ihrer täglichen Arbeit nachgingen, fiel ein Venenkatheter außer Betracht. Zwölfmalige Venenpunktion war ebenfalls nicht zumutbar. Es drängte sich eine praktische, mit wenig Blut (Fingerbeere) arbeitende Methode auf. Die neulich beschriebene Methode nach HUANG und Mitarbeitern (13) verwendet $0,2 \mathrm{~m} /$ oder für Doppelbestimmungen $0,4 \mathrm{~m} /$ Serum. Diese Menge ist gering, aber immer noch zu groß bei Entnahme aus dem Finger. Aus diesem Grunde wurde obige Originalmethode im Maßstab 1:20 in eine Mikromethode transponiert (19). Für eine Doppelbestimmung benötigt man so nur noch $2 \times 10 \mu l$. Die Brauchbarkeit dieser Methode ist durch Vergleichsuntersuchungen gesichert.

\section{Blutentnabme}

Reinigung der Haut mit Åther oder Alkohol. Blutentnahme abwechselnd aus verschiedenen Fingern, zentrifugiert nach genügender Ruhezeit (einige Stdn.) zur Vermeidung von Hämolyse. Für den Einstich wurden die steril verpackten, nach Gebrauch wegwerfbaren Lanzettchen „Hemolet“ von der Fa. Dade Reagents verwendet. Als sehr praktisch hat sich auch eine lokal applizierbare, die Blutungsverhältnisse begünstigende Salbe „Hemo Lube“ erwiesen. (Beides: Merz-Reagentien, Bümpliz/BE).

\section{Entnabmezeiten}

Der Cholesteringehalt wurde stündlich, von $8.00-19.00$ bestimmt. Der Versuch begann morgens $8.00 \mathrm{mit}$ der Bestimmung des Nüchternwertes.

\section{Reagenzien}

1. Standard: $200 \mathrm{mg} / 100 \mathrm{~m} /$ Cholesterin in Eisessig, (Chem. Fabr. Schweizerhall, 1:1 verdünnt; oder Einwägung von Cholesterin p. a. Riedel-de Haen).

2. Vollreagens: $30 \mathrm{~m} /$ Eisessig p. a. (Merck); $60 \mathrm{ml}$ EssigsäureAnhydrid p. a. (Riedel); $10 \mathrm{ml} \mathrm{H}_{2} \mathrm{SO}_{4}$ Konz. p. a. (Merck); $2 \mathrm{~g} \mathrm{Na}_{2} \mathrm{SO}_{4}$ siccum p. a. in Eisbad vermischen, schütteln bis $\mathrm{Na}_{2} \mathrm{SO}_{4}$ (am besten über Nacht) gelöst. - Reagens (1-2 Monate haltbar) im Eiskasten aufbewahren.

\section{Arbeitsgang}

Je $250 \mu l$ Vollreagens werden in 4 saubere, nach Gebrauch wegwerfbare Plastiktuben einpipettiert. Anschließend fügt man Serum usw. nach dem Schema der Tabelle 1 bei.

Tab. 1

\begin{tabular}{|c|c|c|c|}
\hline & $\begin{array}{l}\text { Reagenzien- } \\
\text { Leerwert (RL) }\end{array}$ & Standard & $\begin{array}{l}\text { Analyse (doppelt } \\
\text { bestimmt) }\end{array}$ \\
\hline a) Vollreagens & $250 \mu l$ & $250 \mu l$ & $250 \mu l$ \\
\hline b) Aqua dest. & $10 \mu l$ & - & - \\
\hline c) Standard & - & $10 \mu l$ & - \\
\hline Serum*) & - & - & $10 \mu l$ \\
\hline
\end{tabular}

*) (Serumtropfen an Plastiktubenwand deponieren)

Zum Pipettieren wurden die halb-automatischen Sanz-Mikropipetten verwendet.

Nach dem Verschließen der Tuben werden diese heftig gemischt (Mikromixer sofern vorhanden). Zur Farb-Entwicklung hängt man alle Tuben $20 \mathrm{Min}$. in ein Wasserbad von $24-25^{\circ}$. Es entsteht eine blaugrüne Farbe nach der Liebermann-Burcbard-Reaktion, welche durch den Zusatz von $\mathrm{Na}_{2} \mathrm{SO}_{4}$ beträchtlich stabilisiert wird. Anschließend wird am Mikrospektrokolorimeter (Beckman) bei $610 \mathrm{~m} \mu$ gegen den Reagentien-Leerwert (RL) abgelesen.

$$
\text { Berechnung } \frac{E \text { (Analyse) }}{E \text { (Stand.) }} \cdot 200=\mathrm{mg} \% \text { Cholesterin. }
$$




\section{Ernäbrung und andere Versucbsbedingungen}

$\mathrm{Da}$ die Ernährung und andere Faktoren den Cholesteringehalt (auch Triglyzeride und Lipoproteide) beeinflussen können, steht fest. Nach geomedizinischen Untersuchungen von A. KEYs (14) steht der Cholesterinspiegel in enger Beziehung zum Prozentsatz der Kalorien, den das Fett zur gesamten Kalorienzufuhr beiträgt. Heute wissen wir aber auch, daß man nicht einfach von „Fett" reden darf. Wesentlich ist auch die qualitative Zusammensetzung des Nahrungsfettes. Auch die Unterteilung in tierisches und pflanzliches Fett wird dieser Tatsache nicht gerecht (15). Wir kennen tierische (Schweineschmalz, Butter) und pflanzliche Fette (z. B. Cocosfett) welche die Serumlipoide ansteigen lassen, aber auch tierische (Walöl, Sardinenöle) und pflanzliche (Sonnenblumen- und Maisöl) bei denen sie sinken (15).

Wenn auch in den meisten Arbeiten über Cholesterin, der Ernährung unter den Umweltsbedingungen die größte Aufmerksamkeit geschenkt wird, ist nicht $z u$ vergessen, $d a ß$ es noch andere Faktoren gibt, die zu berücksichtigen sind. Als solche geiten Arbeitsbelastung (17), hereditäre Faktoren (18), psychische Emotionen $(15,18)$. In diesem Zusammenhang ist der gelegentliche Anstieg der Blutlipide infolge psychischer Emotionen während des Hungerversuches bemerkenswert (15). Unter Hungerbedingungen fällt der normale, insbesondere aber der erhöhte Blutlipoidgehalt (16). $\mathrm{Zu}$ diesem Thema haben ferner J. E. Pexerson und Mitarbeiter (19) einen interessanten Beitrag geleistet, auf welchen wir noch zu sprechen kommen. Man darf annehmen, daß auch das Verhältnis der drei Grundnährstoffe Kohlehydrate, Eiweiß und Fett untereinander von Bedeutung für den Cholesterinhaushalt sind (15). Aus diesen Gründen wurde folgende kalorisch austeichende, fettarme, jedoch alle drei Grundnährstoffe enthaltende Nahrung verabreicht, aber nur in beschränkter Quantität, um mögliche Einflüsse niedrig $\mathrm{zu}$ halten:

Friibstück: nach der ersten Blutentnahme etwa $8.30 ; 1$ Tasse Tee gezuckert; 1 Brötchen oder Zwieback mit oder ohne Confiture.

Mittagessen: eingenommen zw. 12-13 Uhr. Grilliertes, mageres Kalbfleisch oder Trockenfleisch, Teigwaren oder Kartoffeln, ohne Fett, Öl od. Butter zubcreitet.

Das Nachtessen wurde erst nach der letzten Blutentnahme eingenommen und ist somit für den Versuch irrelevant.

\section{Ergebnisse}

In den eigenen Versuchen werden unter den besprochenen Versuchsbedingungen einzelne Cholesterin-Tages-

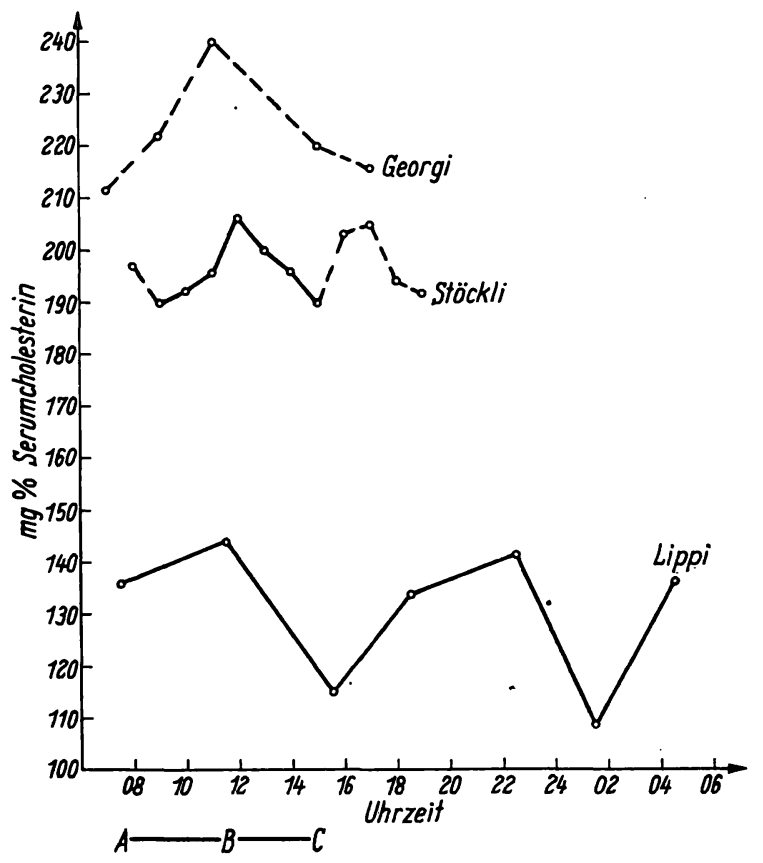

kurven erhalten, die unterschiedlich ausfielen, aber in weiten Abschnitten gleiches oder zumindest ähnliches Verhalten zeigten.

Die Stundenwerte der einzelnen Kurven wurden addiert und gemittelt und in der Abbildung als Summenkurve graphisch dargestellt.

Nach einer leichten Senkung zwischen 8-9 Uhr (als Folge der Nahrungseinnahme?) beginnt die Kurve zu steigen, um zur Mittagszeit einen Höhepunkt (1. Gipfel) zu erreichen. Vom Mittagsgipfel sinken die Cholesterinwerte wieder bis etwa 15 Uhr. In einem nächsten Anlauf erklettern sie den 2. Gipfel (etwa $17 \mathrm{Uhr}$ ) und fallen anschließend erneut ab. Es muß dabei offengelassen werden, ob ähnliche kurzfristige Schwankungen auch über die Nachtzeit fortdauern.

\section{Streubreite der Kurvenwerte:}

Von 136 Doppelbestimmungen ausgehend wurde eine einfache methodische Abweichung von $\mathrm{s}= \pm 3,8 \mathrm{mg} / 100 \mathrm{~m} /$ für eine Einzelbestimmung exrechnet. - Setzt man den methodischen Streubereich wie üblich mit \pm 2 s ein, so heißt dies, daß Einzel-Werte, mehr als $8 \mathrm{mg} / 100 \mathrm{ml}$ vom wahren Wert abweichen, nur noch mit einer Wahrscheinlichkeit von weniger als $5 \%$ zufällig sind. Für unsere Mittelwertkurve aus 15 Probanden ergibt sich somit ein doppelter mittlerer Fehler

$$
2 \mathrm{~s}_{\mathrm{m}}=\frac{2 \mathrm{~s}}{\mathrm{Vn}}= \pm 2 \mathrm{mg} / 100 \mathrm{ml} .
$$

(Bereich, in welchem der Mittelwert mit 95\% Wahrscheinlichkeit streuen kann.)

\section{Diskussion}

Vergleicht man die drei Kurven der Abbildung 1, so fällt eine deutliche Analogie auf. Alle zeigen übereinstimmend einen Anstieg vormittags zum Mittagsgipfel. Die Art und Weise, wie der Anstieg vor sich geht und wo er einsetzt, geben die drei Kurven - ihrem Meßintervall entsprechend - mit verschiedener Genauigkeit an. Aus dem gleichen Grunde sind die Mittagsgipfel zeitlich um eine halbe Stunde verschoben. Diese erweisen sich in der weiteren Gegenüberstellung als eigentliche Umkehrpunkte. Alle drei Kurven zeigen nämlich nach diesem Mittagshoch einen deutlichen Cholesterinabfall, der erst im Verlaufe des mittleren Nachmittages (etwa $15 \mathrm{Uhr}$ ) zum Stillstand kommt.

Bis zu diesem Kurvenabschnitt konnte die vorliegende Arbeit die Befunde von Grorgr und LIPpr im wesentlichen bestätigen; das prinzipiell analoge Verhalten kann nicht übersehen werden. Die quantitativen Unterschiede - die LIPpr-Kurve verläuft durchweg auf einer tieferen Etage - sind durch Anwendung verschiedener chemischer Methoden bedingt. Will man jedoch den Vergleich von diesem Punkt weiterführen, so gelangt man zu keinem positiven Resultat mehr. Das restliche Kurvenstück der Abbildung 1 wurde aus diesem Grunde mit unterbrochener Linie dargestellt. Dieser Teil steht bis zum Erscheinen weiterer bestätigender oder widerlegender Untersuchungen zur Diskussion. Das gleiche gilt für das Gebiet zwischen 8-9 Uhr. Die individuellen Kurven zeigen dort ein unterschiedliches Verhalten. Es gibt solche, die horizontal, abfallend oder ansteigend 
verlaufen; in der Summenkurve ergibt sich ein leichter Abfall. Ebenso interessant wie die Cholesterin-Tagesrhythmik selbst, ist die Größe der dabei auftretenden Schwankungen. Dic folgende Zusammenstellung der Tabelle 2 vermittelt uns einige Anhaltspunkte. Die von GeORGI als Bcispiel gewählte Kurve eines Gesunden (3) weist in der berücksichtigten Zeit eine Schwankung von $28 \mathrm{mg} / 100 \mathrm{ml}$ auf.

Tab. 2

Ausmaß der Cholestcrin-Tages-Schwankung (Werte in $\mathrm{mg} / 100 \mathrm{~m} /$; größtcr minus kleinster Tageswert)

\begin{tabular}{lccc}
\hline \multicolumn{1}{c}{ Autor } & minimal & maximal & Summenkurve \\
\hline Lippr $(24 \mathrm{~h})$ & 20 & 74 & 34 \\
SröckLI $(12 \mathrm{~h})$ & 6 & 66 & 16 \\
\hline
\end{tabular}

Aus den Zahlen der Tabelle 2 geht hervor, daß es Individuen mit schr geringen und solche mit größeren Schwankungen (30\% und mehr) gibt. Möglicherweise sind das die von Peterson (18) als „stable“ oder „labile subjects" bezeichneten Fälle. In den Versuchen von Peterson und Mitarbeitern (19) wurden gesunde Medizinstudenten nach einer längeren Voruntersuchung (mindest. 12 Cholesterinbestimmungen pro Person) bezüglich Konstanz des Cholesteringehalts in sog. „labile subjects" und „stable subjects" eingeteilt. Mit raffinierten Mitteln (akustische Reize, Anwesenheit der Professoren, skin reponse etc.), wurden die Testpersonen in einen emotionellen Spannungszustand hineinmanövriert und dabei die Cholesterinschwankungen verglichen. Die „stable subjects" waren auch unter diesen Bedingungen relativ stabil, während die labile Gruppe z. T. beachtliche Schwankungen aufwies. Ähnliches ergab sich auch unter anderen Bedingungen wie Adrenalininjektionen oder Kälte-Exposition. Die Tatsachen, daß Alter, Geschlecht und Rasse (= Ernährungsunterschied?) ebenfalls von Bedeutung sind, kompliziert die Versuche noch mehr.

Aus diesen und den eigenen Versuchen drängt sich noch eine andere Feststellung auf: Eine zu beliebiger Tages-. zeit ausgeführte Cholesterinbestimmung ist für ein Individuum - ganz besonders, wenn es sich um ein sog. „labiles Subjekt" handelt - nur bedingt repräsentativ. Angaben in der Literatur, die sich bei einzelnen Individuen auf nur eine Cholesterinmessung - möglicherweise noch zu verschiedener Tageszeit vorgenommen stützen, sind dcshalb mit Vorbedacht zu beurteilen. Neben den unter „Ernährung und andere Versuchsbedingungen" erwähnten Faktoren, ist also auch diese Tatsache zu berücksichtigen. Da man im Einzelfall nicht weiß, in welchem Rahmen sich die Schwankungen bewegen, empfiehlt es sich, nur solche Bestimmungen quantitativ miteinander zu vergleichen, die mit gleicher Methode und zur gleichen Tageszeit vorgenommen wurden.

Kommen wir auf die eingangs gestellte Frage zurück, welcher Tagesabschnitt für die Erfassung der absoluten Cholesterinkonzentration und deren charakteristischen Veränderungen der geeignetste sei, so müssen wir diese Frage wie folgt beantworten: Typisch für alle Normalpersonen ist offenbar das Maximum der Mittagszeit. Der Wert um 15 Uhr stellt bei den meisten Personen ein Minimum dar, wclches deutlich niedriger liegt als die um $8 \mathrm{Uhr}$ gemessenen Werte. Eine optimale Erfassung des Cholesterinspiegels im Serum, seiner Schwankungsrichtung und -breite wäre demnach durch eine dreifache Bestimmung um 8, 12 und $15 \mathrm{Ubr}$ gewährleistet. Mit der Fingerbeerenmethode ist heute eine solche Forderung in der Klinik ohne Weiteres zu erfüllen.

Nach allem scheint es nunmehr von Interesse, entsprechende Untersuchungen unter pathologischen Verhältnissen durchzuführen. Dabei könnten womöglich die Resultate noch signifikanter gestaltet werden, sofern man - wie dies seinerzeit auch GEORGI (3) getan hat statt der gewöhnlichen Bestimmungen einen Belastungstest durchführt.

Herrn Prof. F. Georgr, der inzwischen leider verstorben ist, gebührt mein bester Dank für die wertvolle Unterstützung und Kritik der vorliegenden Arbeit. Für praktische Ratscbläge bei der Ausführung der Untersuchungen und für statistische Berechnung der Ergcbnisse bin ich Herrn Dr. H. P. RIEDER zu Dank verpflichtet.

\section{Literatur}

1. Heyman, A., M. D. Nefzger und E. H. Estes, Arch. Neurol. Psychiatr., Chicago 5, 264 (1961). - 2. GeorGr, F., Fortschr. Ncurol. 6, 340 (1934). - 3. Georgr, F., Schweiz. med. Wschr. 20, 539 (1944). - 4. Stenberg, S., Acta med. Scand. 71, 1 (1929). 5. SaArnio, L., Sonderdruck aus Acta Soc. med. Fenn. Duodecim, B $26 / 1$ (1938). - 6. Ederle, W., Jvers. Dtsch. Neurol. Psychiatr., Dresden (1935). - 7. BruN, G., Acta psychiatr. neurol., K'hvn, Suppl. Bd. 2 (1936): Changes in Lipide Contents of serum in Patients with Manic-Depressive. Psychosis. Copenhagen-Iondon (1940). - 8. GildeA, R., Arch. Neurol. (Amer.) 43, 932 (1940), zit. nach Georgi, F. (3). - 9. Sperry, W. M., J. biol. Chemistry 117, 391 (1937). - 10. Boyd, E. N. I., J. biol. Chemistry 110, 61 (1935).
-11. Turner, K. B. und Steiner A., J. Clin. Invest. 18, 45 (1939) -12. Lippi, M. und L. Argroles, Minerva med. 41, 307 (1950). 13. Huang, T. C., C. P. Chen, Vera Wefler and Alan Rafterr, Analytic. Chem. 33, 1405 (1961). - 14. Keys, A., J. Amer. Med. Ass. 164, 1912 (1957). - 15. Schettler, G. und M. Eggstein, Dtsch. med. Wschr. 16, 702 (1958); 17, 750 (1958). - 16. KeMPNER, W., Ann. Int. Med. 31, 821 (1949). - 17. ManN, G. V., Teel C., Hayes O., McNally A. and Bruno D., N. England J. Med. 253, 349 (1955). - 18. Petierson, J. E. A. A. Wilcox, M. I. Haley and R. A. Kertri, Circul. 22, 247 (1960). - 19. Rieder, H. P., Laborinterne Vorschrift (1962). - 20. Berenstein, S. und F. Georgr, Schwciz. med. Wschr. 75, 710 (1945).
Dr. Charles Stöckli Forschungslab. der Neurolog. Univcrsitätsklinik Basel (Schwciz) 